\title{
(6) OPEN ACCESS \\ Sectorial loss of retinal ganglion cells in inherited photoreceptor degeneration is due to RGC death
}

\author{
Diego García-Ayuso, ${ }^{1}$ Manuel Salinas-Navarro, ${ }^{1}$ Francisco Manuel Nadal-Nicolás, ${ }^{2}$ \\ Arturo Ortín-Martínez, ${ }^{1}$ Marta Agudo-Barriuso, ${ }^{2}$ Manuel Vidal-Sanz, ${ }^{1}$ \\ María P Villegas-Pérez ${ }^{1}$
}

\begin{abstract}
${ }^{1}$ Laboratorio de Oftalmología Experimental, IMIB, Facultad de Medicina, Universidad de Murcia, Espinardo, Murcia, Spain

2Unidad de Investigación, Hospital Universitario Virgen de la Arrixaca, IMIB Servicio Murciano de Salud, Fundación para la Formación e Investigación Sanitarias de la Región de Murcia, El Palmar Murcia, Spain
\end{abstract}

\section{Correspondence to}

Professor María P VillegasPérez, Laboratorio de Oftalmología Experimental Facultad de Medicina, Universidad de Murcia, Campus de Espinardo, Espinardo, Murcia 30100, Spain; mpville@um.es

Received 3 July 2013 Revised 10 October 2013 Accepted 17 November 2013 Published Online First 10 December 2013

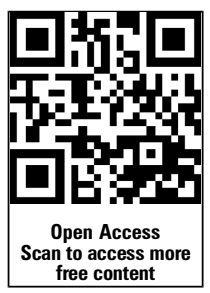

CrossMark

To cite: García-Ayuso D, Salinas-Navarro M, NadalNicolás FM, et al. $\mathrm{Br}$ J Ophthalmol 2014:98:396401.

\section{ABSTRACT}

Aims To investigate the cause of retinal ganglion cell (RGC) loss in dystrophic aged Royal College of Surgeons (RCS) rats.

Methods RCS- $p+$ (dystrophic) female rats of postnatal times (P365, P450 and P540) and age-matched RCS-p1 $r d y+$ (non-dystrophic) rats were used. In whole-mounted retinas, RGCs were doubly labelled with Fluorogold (FG) retrogradely transported from the superior colliculi and Brn3a immunohistochemistry. RGC axons were labelled with anti-neurofilament antibodies. Automatic image analysis techniques allowed quantification of the total population of RGCs per retina and construction of isodensity maps to investigate RGC topology.

Results Dystrophic retinas showed at all times studied wedge-shaped sectors devoid of $\mathrm{FG}^{+}$and $\mathrm{Brn} \mathrm{a}^{+} \mathrm{RGCs}$. These sectors were also devoid of neurofilament-labelled axons. The total number of $\mathrm{FG}^{+} \mathrm{RGC}$ and $\mathrm{Brn} 3 \mathrm{a}^{+} \mathrm{RGC}$ per retina was significantly smaller in dystrophic rats at P540, revealing RGC death at this age. The total number of $\mathrm{FG}^{+} \mathrm{RGCs}$ was smaller than those of $\mathrm{Brn3a}^{+} \mathrm{RGCS}$ at P540, indicating a disturbance of the retrograde axonal transport at this age.

Conclusions RGC double labelling documents that sectorial RGC loss in aged dystrophic RCS rats is mainly due to RGC death, although a deficit of the retrograde axonal transport exists also at the more advanced ages.

\section{INTRODUCTION}

Inherited human retinal photoreceptor degenerations comprise a large group of diseases that lead to blindness. These diseases, which are very heterogeneous, affect 1 in 3000 individuals and are one of the main causes of blindness in working-age individuals. ${ }^{1}$ The most common form of inherited retinal photoreceptor degeneration is retinitis pigmentosa (RP), affecting 1 in every 4000 individuals. $^{2}$ There are various forms of this disease, but in many of them there is primary degeneration of rods. The disease is usually monogenic, although more than 200 locus have been described, ${ }^{12}$ and the mutations affect photoreceptor or retinal pigment epithelium (RPE) proteins involved in different retinal functions. ${ }^{1}$ During the course of the disease, one of the commonest clinical signs is optic nerve pallor that has been recently shown to be due to retinal ganglion cell (RGC) degeneration. ${ }^{3}$ Why do RGCs die in RP is still a matter of discussion, but it is important to know the underlying reasons in order to design strategies for the treatment of the disease.
Animal models have been useful for studying the mechanisms of inherited retinal degenerations. In certain cases, the mutations observed in humans also cause retinal degeneration in animals, and thus the animal models faithfully reproduce the disease. ${ }^{4}$ During the past 20 years, and in order to understand the mechanism of RGC degeneration in inherited photoreceptor degenerations, we have investigated the dynamics of retinal degeneration in various animal models. Our studies have shown that Royal College of Surgeons (RCS) ${ }^{5-7}$ and $\mathrm{P} 23 \mathrm{H}-1$ rats $^{8}$ and $\mathrm{rd}-1$ mice ${ }^{9}$ show a certain degree of RGC loss during the course of the disease. We have shown that RGC loss occurs in triangular sectors that extend from the optic disc to the retinal periphery and we have also reported the presence of these wedge-like sectors lacking RGCs in animal models of light-induced retinal degeneration. ${ }^{10}{ }^{11}$ In these models, we have documented that RGC loss is a consequence of RGC axonal compression by the inner retinal vessels, which are displaced and surrounded by retinal pigment epithelial cells. ${ }^{6} 71011$ In our previous studies ${ }^{5-7}$ RGC loss was documented using retrogradely transported neuronal tracers and thus it could be argued that the absence of retinal neurons reflected axonal transport impairment. In the present study, we have used for the first time double-labelling techniques as well as automatic image analysis routines to document unequivocally that sectorial RGC loss in RCS rats is indeed due to RGC death.

\section{MATERIALS AND METHODS}

Aged RCS- $p$ 1- (dystrophic) female rats of postnatal times (P365, P450 and P540) and age-matched RCS- $p 1$ rdy1- (non-dystrophic) female rats were used for this study. Animals were obtained from the breeding colony of the University of Murcia (Murcia, Spain) and housed in temperaturecontrolled and light-controlled rooms with a $12 \mathrm{~h}$ light/dark cycle (light from 08:00 to 20:00) and had food and water ad libitum. Light intensity within the cages ranged from 5 to 30 lux. Animal manipulations were carried out following the Spanish and European Union regulations for the use of animals in research (Council Directive 86/ 609/EEC) and the ARVO statement for the use of animals in ophthalmic and vision research.

Surgical manipulations were carried out under general anaesthesia induced with an intraperitoneal injection of a mixture of ketamine $(70 \mathrm{mg} / \mathrm{kg}$, Ketolar, Parke-Davies, S.L., Barcelona, Spain) and xylazine $(10 \mathrm{mg} / \mathrm{kg}$, Rompún, Bayer, S.A., Barcelona, 
Spain). For recovery from anaesthesia, rats were placed in their cages and an ointment containing tobramycin (Tobrex, Alcon S.A., Barcelona, Spain) was applied on the cornea to prevent corneal desiccation. Animals were sacrificed by an intraperitoneal injection of an overdose of sodium pentobarbital (Dolethal Vetoquinol, Especialidades Veterinarias, S.A., Alcobendas, Madrid, Spain).

\section{Horseradish peroxidase injection}

In some animals, a solution of type I horseradish peroxidase (HRP, $44 \mathrm{kDa}$, Sigma P 8125, 12000 units, $143 \mathrm{mg} / \mathrm{mL}$ in sterile saline) was injected in the femoral vein $15 \mathrm{~min}$ before processing to label the retinal blood vessels, as previously described. 61011

\section{Retinal ganglion cells retrograde labelling from the superior colliculi}

Fluorogold (FG) was applied to both superior colliculi (SCi), which are the main retinorecipient target regions in the brain, 1 week before animal processing to retrogradely label the RGCs, following previously described methods. ${ }^{6}$ 10-12

\section{Tissue processing}

Rats were perfused transcardially through the ascending aorta first with saline and then with $4 \%$ paraformaldehyde in $0.1 \mathrm{M}$ phosphate buffer ( $\mathrm{pH} 7.4$ ).

\section{Whole-mount preparations}

Twenty dystrophic RCS animals with FG applied in the SCi were processed at P365 $(n=7)$, P450 $(n=6)$ or P540 $(n=7)$. As controls, seven FG-traced non-dystrophic RCS rats were processed at P365 $(n=4)$ or at P540 $(n=3)$. After perfusion, eyes were enucleated and retinas dissected as whole mounts. ${ }^{8} 10$

\section{Histochemistry}

Some retinas were reacted for HRP demonstration using the Hanker-Yates technique as previously described. ${ }^{5} 6$ 9-11

\section{Immunohistofluorescence}

Retinas were incubated with an antibody against Brn3a (goat anti-Brn3a, C-20, Santa Cruz Biotechnologies, Heidelberg, Germany) to identify RGCs, as previously reported. ${ }^{8}{ }^{10}$ Phosphorylated high-molecular-weight subunits of the neurofilament triplet ( $\mathrm{pNFHs)}$ were then immunodetected by incubation with the RT97 antibody (mouse anti-pNFH, Hybridoma Bank, University of Iowa, Iowa, USA) to label the RGC axons in the retina, following standard procedures in our laboratory. 5681011

\section{Retinal image analysis}

Retinal whole mounts were examined and photographed under a fluorescence microscope (Axioscop 2 Plus; Zeiss Mikroskopie, Jena, Germany) following standard procedures in our laboratory. ${ }^{8} \quad 10$ The microscope is equipped with various filters, a digital high-resolution camera (ProgResTM C10, Jenoptik, Jena, Germany) and a computer-driven motorised stage (Pro-ScanTM H128 Series, Prior Scientific Instruments, Cambridge, UK) controlled by IPP (IPP V.5.1 for Windows; Media Cybernetics, Silver Spring, Maryland, USA) with a microscope controller module (Scope-Pro V.5.0 for Windows; Media Cybernetics, Silver Spring, Maryland, USA). To make reconstructions of retinal whole mounts, retinal multiframe acquisitions were acquired in a raster scan pattern using a $\times 10$ objective (Plan-Neofluar, 10x/0.30; Zeiss Mikroskopie, Jena, Germany). Single frames were focused manually. The images taken for each whole mounted retina were combined into a single high- resolution composite image of the whole retina using IPP and further processed using Adobe Photoshop CS 5.0.1 (Adobe Systems, Inc., San Jose, California, USA) when needed.

\section{RGC counting}

The individual fluorescent images taken in each retina were processed by a specific cell counting subroutine developed by our group to count $\mathrm{FG}^{+}$or Brn $3 \mathrm{a}^{+}$RGCs. Briefly, we used the IPP macro language to apply a sequence of filters and transformations to each image in order to clarify cell limits and separate individual cells for automatic cell counting. These procedures have been previously reported in detail. ${ }^{8} 1012$

\section{Isodensity maps}

To demonstrate the spatial distribution of both $\mathrm{FG}^{+}$and $\mathrm{Brn}_{3} \mathrm{a}^{+}$ RGCs, we constructed an isodensity map for each retina using the specific subroutine developed in our laboratory. ${ }^{8} \quad 10 \quad 12$ Briefly, using IPP macro language every frame was divided into 64 sampling areas, in which RGC counts were obtained and cell densities calculated. These densities were represented as filled contour plots using SigmaPlot (SigmaPlot V.9.0 for Windows; Systat Software, Inc., Richmond, CA, EEUU).

\section{Statistics}

Statistical analysis was done using SigmaStat V.3.1 for Windows (SigmaStat for Windows V.3.11; Systat Software, Inc,). KruskalWallis test was used when comparing more than two groups, and Mann-Whitney test was used when comparing two groups only. Differences were considered significant when $\mathrm{p}<0.05$.

\section{RESULTS}

In this study, RGCs have been double labelled with two different methods in order to investigate RGC loss in the RCS rat retina. FG labelling allows visualisation of the RGCs retrogradely traced from the $\mathrm{SCi}$ while Brn3a immunodetection labels surviving RGCs independently of their axonal transport capabilities. ${ }^{12}$ Both markers allowed us to observe in wholemount preparations the distribution across the retina of the entire RGC population (figure 1).

Non-dystrophic rat retinas presented a typical distribution of RGCs and a normal appearance of the nerve fibre layer (data not shown; for a review, see ref. 6). Dystrophic retinas showed at all times studied wedge-shaped sectors devoid of both $\mathrm{FG}^{+}$ and $\mathrm{Brn}_{3 \mathrm{a}^{+} \mathrm{RGCs}}$ (figure 1), allowing us to conclude that within these sectors RGCs have died. Within the sectors, but also all throughout the entire retina, there were also some Brn3a $\mathrm{a}^{+}$RGCs that were not labelled with FG (figure $1 \mathrm{H}, \mathrm{I}$ ), suggesting that these surviving RGCs were not able to transport FG retrogradely. The wedge-shaped sectors were also devoid of neurofilament-labelled $\left(\mathrm{pNFH}^{+}\right)$axons (figure 1) and were observed at earliest times analysed in the ventral retina but later spread dorsally (figure 2; for a review, see ref. 6). The $\mathrm{pNFH}^{+}$ axons also showed multiple areas of axonal compression by retinal vessels (figure $3^{5}{ }^{6}$ ). We have previously shown that axonal transport is interrupted in the areas of axonal strangulation and proposed that this alteration finally causes axonal transection. ${ }^{5}{ }^{6-11}$ Near the strangulation points, some RGC bodies whose axons were affected became also intensely $\mathrm{pNFH}^{+}$ (figure 3). ${ }^{5} 6^{6-10}$ These areas of axonal strangulation could be seen often at the vertex of the sectors devoid of RGCs and $\mathrm{pNFH}^{+}$axons (figures $1 \mathrm{~F}, \mathrm{G}$ and $3 \mathrm{~A}, \mathrm{C}$ ).

The total number of $\mathrm{FG}^{+}$RGCs and Brn3a ${ }^{+}$RGCs in the right and left retinas of non-dystrophic and dystrophic animals processed at different survival intervals can be observed in table 1 

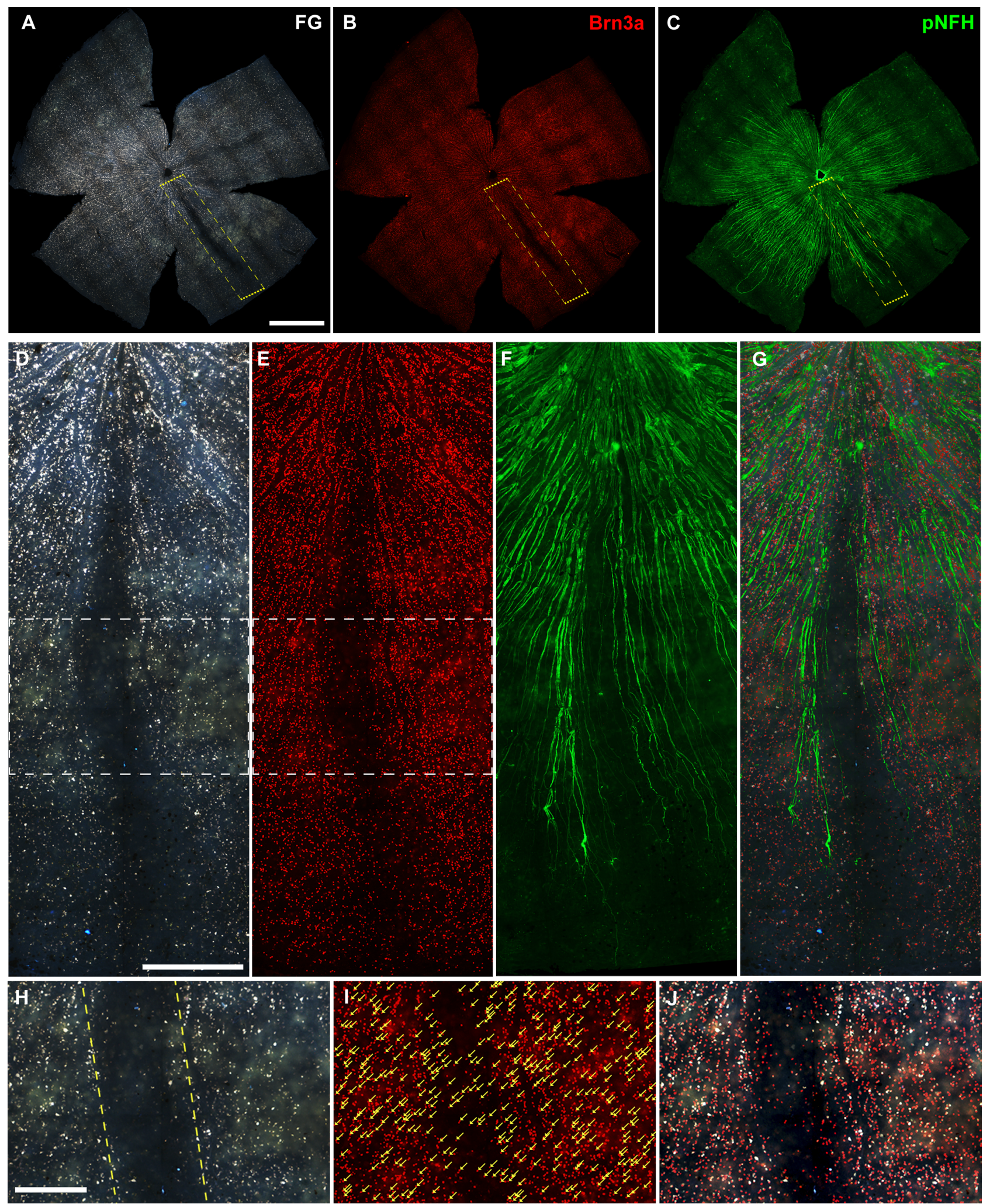

Figure 1 Sector of retinal ganglion cell (RGC) loss in the Royal College of Surgeons (RCS) rat. Photomontages taken with different fluorescence filters of a representative right retina from a P540 RCS rat showing FG ${ }^{+} R G C s(A)$, Brn3a ${ }^{+} R G C s(B)$ and pNFH ${ }^{+}$axons (C). An inferonasal sector devoid of both RGCs and pNFH+ axons can be seen (A-C). This same sector can be seen at a higher magnification in (D-G). (G) is a merged image that includes (D), (E) and (F). The areas highlighted in (D) and (E) can be seen at a higher magnification in $(H)$ and $(I)$, and $(J)$ is a merged image that contains $(\mathrm{H})$ and $(\mathrm{I})$. In (I), the Brn3a ${ }^{+}$cells that were not labelled with FG have been marked with an arrow. Scale bar: A-C=1 mm; D$\mathrm{G}=50 \mu \mathrm{m} ; \mathrm{H}-\mathrm{J}=25 \mu \mathrm{m}$. FG, Fluorogold.

and figure 4. There was no difference (Mann-Whitney test)

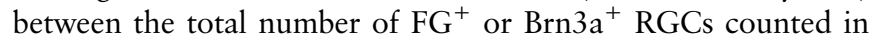
the left and right retinas processed at any given time point.
However, when we compared the total number of $\mathrm{FG}^{+}$or

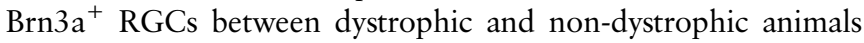
at the ages analysed, we found that dystrophic animals had 

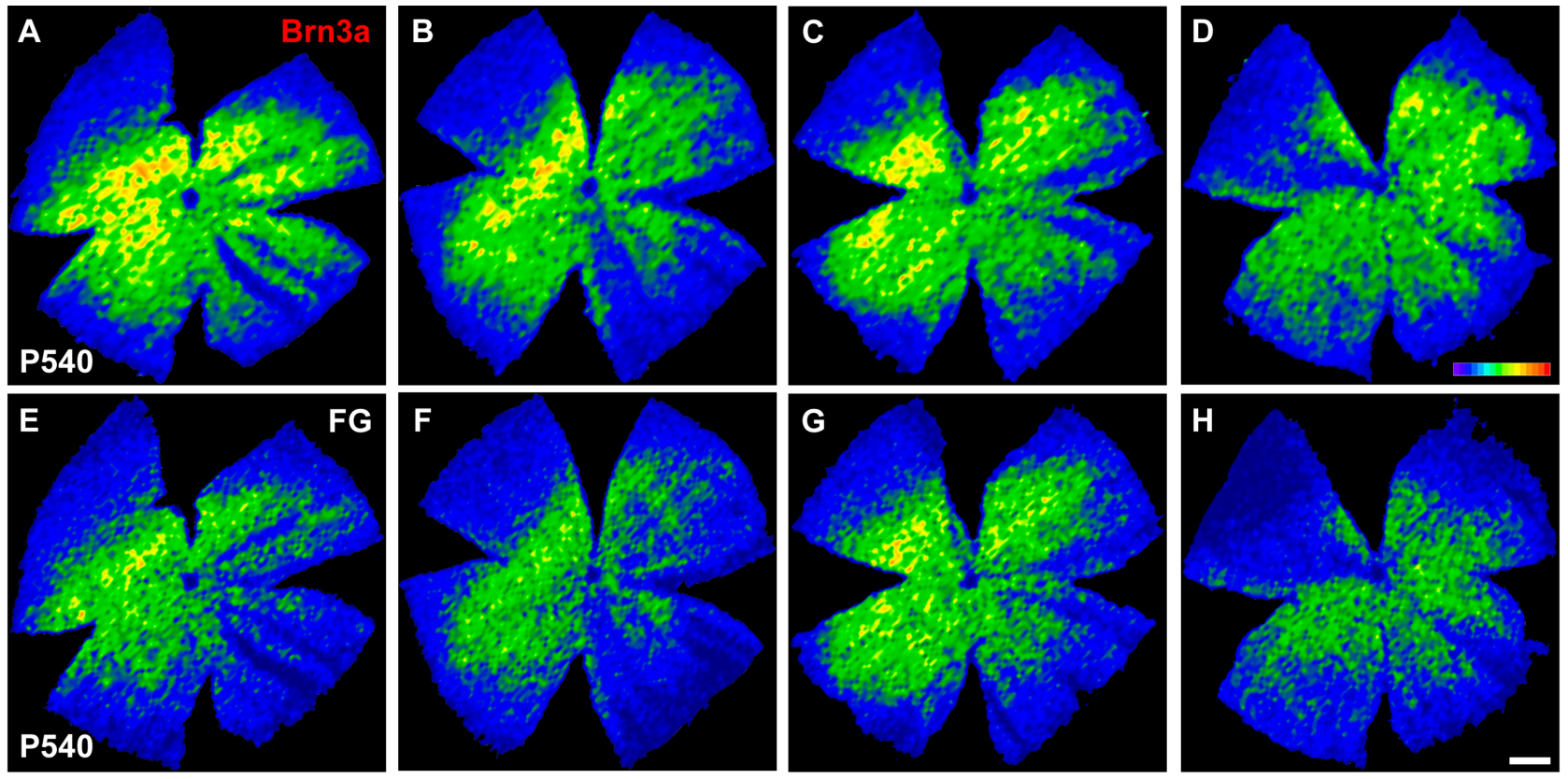

Figure 2 Isodensity maps. Isodensity maps obtained from whole-mounted retinas (see 'Methods' section) that reveal sectors devoid of both FG ${ }^{+}$

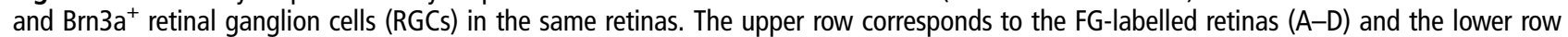
corresponds to the same retinas but immunoreacted for Brn3a (E-H). RGC density values follow a colour-scale range (shown in $\mathrm{D}$ ) goes from 0 (purple) to 3500 or higher (red) RGCs/mm². Scale bar=1 mm. FG, Fluorogold.

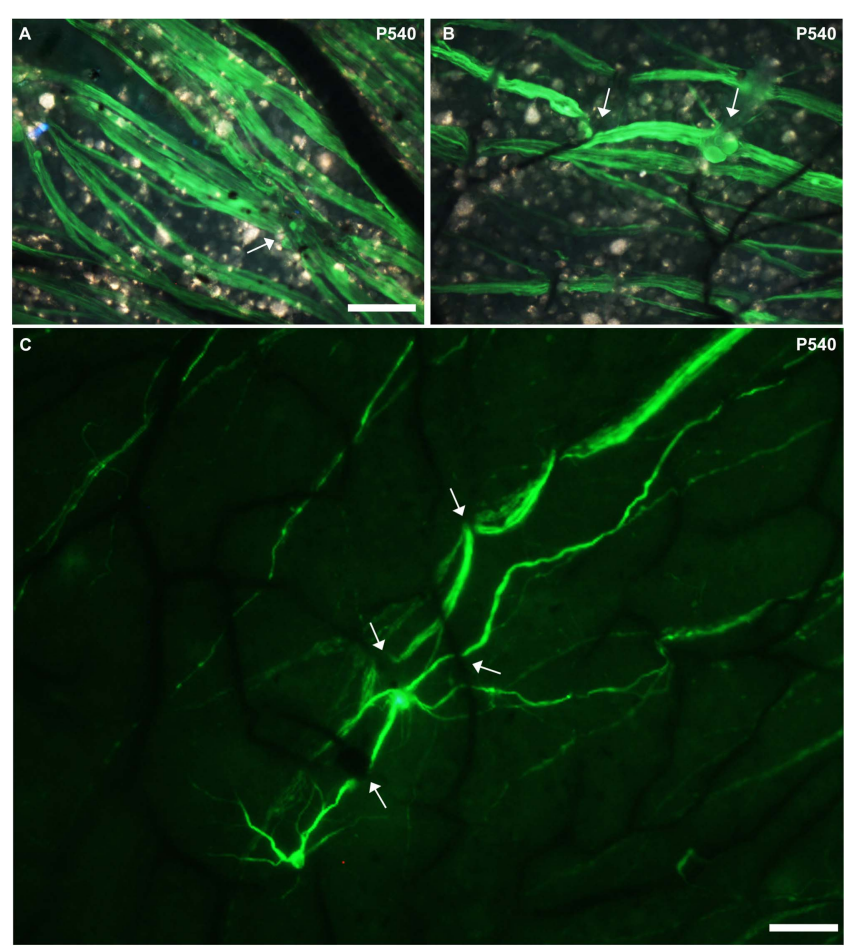

Figure 3 Retinal ganglion cell (RGC) axonal strangulations.

Photomicrographs from whole-mounted retinas of P540 Royal College of Surgeons rats. pNFH ${ }^{+}$axons appear in green and vessels in black (horseradish peroxidase labelling). In (A), an axonal strangulation (arrow) can be seen at the beginning of a sector lacking RGC somas and axons. In (B), axonal strangulations (arrow) and axonal bulbs (arrow) can be observed. In (C), some axonal strangulations (arrow) can be seen close to $\mathrm{pNFH}^{+} \mathrm{RGC}$ bodies. Scale bar $=50 \mu \mathrm{m}$. smaller numbers at the oldest age studied (P540, table 1, figure 4, Mann-Whitney test). Also, the number of $\mathrm{FG}^{+}$or Brn3a ${ }^{+}$RGCs decreased with age in dystrophic animals because at P540 these animals had significantly smaller numbers than at earlier ages (table 1, figure 4, Kruskal-Wallis test).

Because both the number of $\mathrm{FG}^{+}$and $\mathrm{Brn}_{3} \mathrm{a}^{+}$RGCs decreased significantly with age, we conclude that RGCs die with age in these retinas. The number of $\mathrm{Brn}_{3} \mathrm{a}^{+} \mathrm{RGCs}$ is always higher than the number of $\mathrm{FG}^{+}$RGCs both in normal animals ${ }^{12}$ and in the non-dystrophic and dystrophic animals of this study (table 1). These differences do not reach statistical significance in normal animals ${ }^{12}$ or in the non-dystrophic animals in this study (table 1). However, in the dystrophic animals, we found that the number of $\mathrm{FG}^{+} \mathrm{RGCs}$ was significantly smaller than the

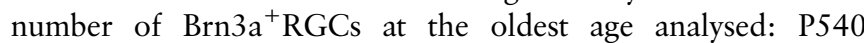
(table 1, figure 4, Mann-Whitney test, $\mathrm{p}<0.05$ ). Because FG is a retrogradely transported tracer and Brn3a is a structural component of RGCs, and because there were always some surviving Brn $3 \mathrm{a}^{+} \mathrm{RGCs}$ in the sectors lacking $\mathrm{FG}^{+} \mathrm{RGCs}$, we conclude that RCS rats show a deficit of the retrograde axonal transport at the oldest age analysed that may precede RGC loss. Because

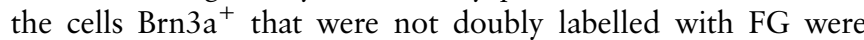
located not only within the sectors but all throughout the retina (figure $1 \mathrm{H}, \mathrm{I}$ ), it is possible that the alteration of the retrograde axonal transport is not only restricted to the sectors.

\section{DISCUSSION}

This study uses a double-labelling technique for the first time to identify RGCs in dystrophic RCS rats to document unequivocally that the lack of retrogradely labelled RGCs in retinal sectors is mainly due to RGC loss. ${ }^{5-7}$ We use two different specific RGC labelling methods-retrograde tracing with FG and 
Table 1 Total number of FG and Brn3a labelled RGCs per retina in non-dystrophic and dystrophic RCS rats at different postnatal days

\begin{tabular}{|c|c|c|c|c|c|c|c|c|c|c|}
\hline \multirow{4}{*}{$\begin{array}{l}\text { Marker } \\
\text { Rat }\end{array}$} & \multicolumn{4}{|c|}{ Non-dystrophic RCS } & \multicolumn{6}{|c|}{ Dystrophic RCS } \\
\hline & \multicolumn{10}{|l|}{ FG } \\
\hline & \multicolumn{2}{|l|}{ P365 } & \multicolumn{2}{|l|}{ P540 } & \multicolumn{2}{|l|}{ P365 } & \multicolumn{2}{|l|}{ P450 } & \multicolumn{2}{|l|}{ P540 } \\
\hline & Left & Right & Left & Right & Left & Right & Left & Right & Left & Right \\
\hline 1 & 81029 & 78002 & 74143 & 75380 & 69755 & 78323 & 80481 & 73921 & 42402 & 57045 \\
\hline 2 & 75922 & 77757 & 80382 & 73654 & 75329 & 69007 & 60612 & 68336 & 39736 & 54304 \\
\hline 3 & 79032 & 72202 & 76983 & 79860 & 72891 & 69087 & 72451 & 68672 & 60892 & 42580 \\
\hline 4 & 75326 & 81205 & & & 76047 & 69045 & 80007 & 81416 & 56217 & 52624 \\
\hline 5 & & & & & 70837 & 66010 & 60612 & 68336 & 61549 & 54714 \\
\hline 6 & & & & & 76732 & 65554 & 67695 & 62501 & 74494 & 66754 \\
\hline 7 & & & & & 67254 & 69174 & & & 55126 & 55730 \\
\hline Mean \pm SD & $77827 \pm 2323$ & $77291 \pm 3238$ & $77169 \pm 2550$ & $76298 \pm 2615$ & $72692 \pm 3299$ & $69457 \pm 3897$ & $70309 \pm 8134$ & $70530 \pm 5881$ & $55730 \pm 11000$ & $54735 \pm 6571$ \\
\hline Mean \pm SD & $77599 \pm 2830$ & & $76733 \pm 2619$ & & $71074 \pm 3956$ & & $70420 \pm 7098$ & & $55232 \pm 9074^{*} \dagger$ & \\
\hline
\end{tabular}

\begin{tabular}{|c|c|c|c|c|c|c|c|c|c|c|}
\hline \multirow{4}{*}{$\begin{array}{l}\text { Marker } \\
\text { Rat }\end{array}$} & \multicolumn{4}{|c|}{ Non-dystrophic RCS } & \multicolumn{6}{|c|}{ Dystrophic RCS } \\
\hline & \multicolumn{10}{|l|}{ Brn3a } \\
\hline & \multicolumn{2}{|l|}{ P365 } & \multicolumn{2}{|l|}{ P540 } & \multicolumn{2}{|l|}{ P365 } & \multicolumn{2}{|l|}{ P450 } & \multicolumn{2}{|l|}{ P540 } \\
\hline & Left & Right & Left & Right & Left & Right & Left & Right & Left & Right \\
\hline 1 & 81074 & 79849 & 80305 & 80190 & 75129 & 74315 & 83619 & 79597 & 74524 & 59893 \\
\hline 2 & 80607 & 83165 & 78259 & 79158 & 77454 & 81118 & 71323 & 79788 & 71523 & 71562 \\
\hline 3 & 80523 & 78892 & 79126 & 78950 & 72605 & 69637 & 69905 & 78511 & 66819 & 69345 \\
\hline 4 & 78224 & 78892 & & & 78564 & 71368 & 79785 & 71323 & 68878 & 69361 \\
\hline 5 & & & & & 75915 & 82194 & 76037 & 76937 & 71895 & 69384 \\
\hline 6 & & & & & 81530 & 81456 & 76657 & 73228 & 77744 & 72906 \\
\hline 7 & & & & & 81223 & 78392 & & & 71134 & 70838 \\
\hline Mean \pm SD & $80107 \pm 1107$ & $80199 \pm 1756$ & $79230 \pm 838$ & $79432 \pm 542$ & $77488 \pm 3007$ & $76925 \pm 4763$ & $76221 \pm 4682$ & $76564 \pm 3.217$ & $71788 \pm 3308$ & $69041 \pm 3937$ \\
\hline Mean \pm SD & $80153 \pm 1468$ & & $79331 \pm 713$ & & $77207 \pm 3993$ & & $76392 \pm 4020$ & & $70414 \pm 3887^{*}$ & \\
\hline
\end{tabular}

immunodetection of Brn3a to identify RGCs-and we show

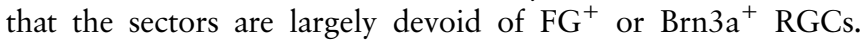
From our previous work in the rat retina, we know that immunodetection of Brn3a is a very good labelling technique that does not depend on axonal transport to identify RGCs in the normal and injured retinas, ${ }^{12}$ and therefore we exclude an alteration of the axonal transport as the main cause of the absence of $\mathrm{FG}^{+} \mathrm{RGCs}$ in the sectors. On the other hand, we also show in dystrophic animals that the number of Brn $3 \mathrm{a}^{+} \mathrm{RGCs}$ in the sectors (and in the retinas) was always higher than the number of $\mathrm{FG}^{+} \mathrm{RGCs}$, and this difference was significant at the more advanced age studied, P540, indicating that at least at this time point some RGCs have lost their capacity for normal retrograde transport. Previous studies in RCS rats have also shown an alteration of the retrograde axonal transport ${ }^{13}$ and a decline of dendritic transport and regenerative capacities of the RGCs. ${ }^{14}$ However, other authors have not found RGC death or alterations of the axonal transport in aged RCS rats, ${ }^{15}$ but these studies have used younger animals than we used and thus it is possible that the discrepancies between the different studies are due to the differences in the methodology employed.

The RCS rat suffers an inherited photoreceptor degeneration $^{16}$ caused by a recessive mutation of the MERTK gene that prevents RPE phagocytosis. ${ }^{17}$ RCS rats are a good animal model of RP because they show signs that are similar to those seen in
RP patients, namely intraretinal pigmentation, blood vessel thinning and optic nerve paleness. ${ }^{67}$ How a disease of the outer retina could affect the optic nerve has been the subject of controversy for a long time. ${ }^{2}$ Although some early studies found a decreased number of RGCs in RP patients, ${ }^{18}$ it has not been until recently that RGC loss has been clearly documented. ${ }^{3} 19$ Why do RGCs die in the course of RP is unknown, but transneuronal degeneration after photoreceptor loss or vascular occlusion has been proposed as the possible mechanism. ${ }^{20}$ Indeed, localised sectorial nerve fibre defects have been observed in outer retinal degenerations, ${ }^{21}$ and RP patients show a decrease in thickness of the retinal nerve fibre layer. ${ }^{19}$ Here we document that in the RCS rat the nerve fibre layer shows sectorial deficits and that there is RGC death in triangular sectors of the retina. We have previously shown that RGC axonal compression by retinal vessels is a phenomenon common to this and other animal models of photoreceptor degeneration, ${ }^{8} 911$ and therefore it is tempting to suggest that this is also the cause of RGC degeneration in RP. Localised sectorial RGC loss is not unique to diseases of the outer retina. Indeed, it is a common phenomenon observed in other retinal and optic nerve diseases such as glaucoma and optic neuropathy, ${ }^{22} 23$ as well as in animal models of these diseases, ${ }^{12}$ in systemic pathologies such as arterial hypertension and also in some normal patients. ${ }^{24}$ Axonal vascular compression has been proposed also 


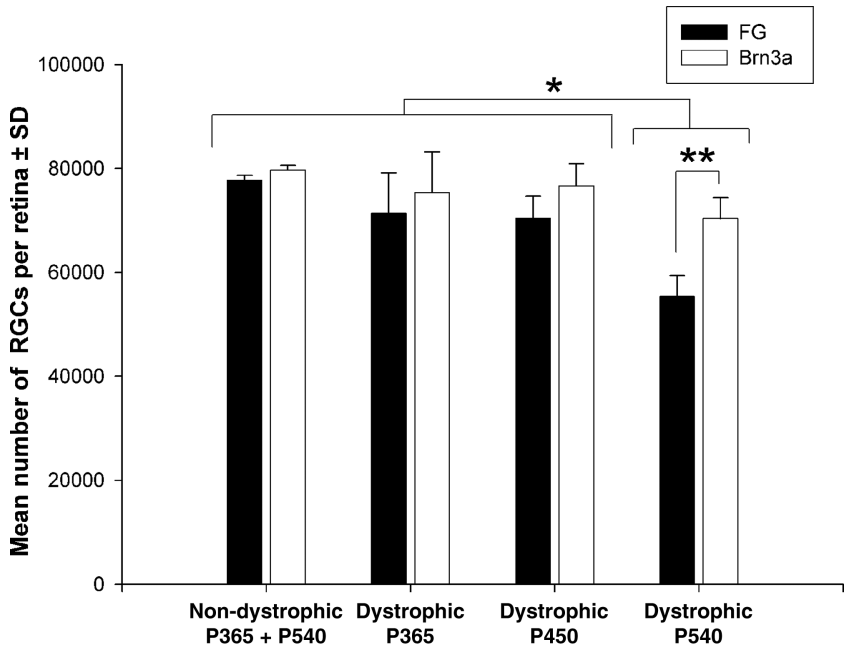

Figure 4 Population of retinal ganglion cells (RGCs) in the Royal College of Surgeons (RCS) rat. Mean numbers $( \pm \mathrm{SD})$ of $\mathrm{FG}^{+}$and Brn3a ${ }^{+} R G C s$ in non-dystrophic and dystrophic RCS rats at different postnatal $(\mathrm{P})$ times. ${ }^{*} \mathrm{FG}$ and Brn3a numbers are significantly lower in dystrophic RCS rats at P540. ${ }^{* *} F$ numbers are significantly lower than the Brn3a numbers obtained in the same retinas. FG, Fluorogold.

as the cause of diseases of the central nervous system. ${ }^{25}$ We would like to propose that axonal vascular compression may be a more frequent than thought pathogenetic mechanism of disease.

Contributors DG-A, MS-N, FMN-N, AO-M and MPV-P were responsible for conception and design, or analysis and interpretation of data. DG-A, MA-B, MV-S and MPV-P were responsible for drafting the article or revising it critically for important intellectual content. All authors approved the final version of the manuscript to be published.

Funding Spanish Ministry of Economy and Competitiveness ISCIII-FEDER: PI13/ 01266, PI13/00643, PI10/01496, PI10/00187, FIS PS0901854, SAF-2012-38328, RD12/0034/0014. Fundación Séneca de la Región de Murcia: 04446/GERM/07.

\section{Competing interests None.}

Provenance and peer review Not commissioned; externally peer reviewed.

Open Access This is an Open Access article distributed in accordance with the Creative Commons Attribution Non Commercial (CC BY-NC 3.0) license, which permits others to distribute, remix, adapt, build upon this work non-commercially, and license their derivative works on different terms, provided the original work is properly cited and the use is non-commercial. See: http://creativecommons.org/ licenses/by-nc/3.0/

\section{REFERENCES}

1 Wright AF, Chakarova CF, Abd El-Aziz MM, et al. Photoreceptor degeneration: genetic and mechanistic dissection of a complex trait. Nat Rev Genet 2010;11:273-84.
2 Hartong DT, Berson EL, Dryja TP. Retinitis pigmentosa. Lancet 2006:368:1795-809.

3 Eng JG, Agrawal RN, Tozer KR, et al. Morphometric analysis of optic nerves and retina from an end-stage retinitis pigmentosa patient with an implanted active epiretinal array. Invest Ophthalmol Vis Sci 2011;52:4610-16.

4 Chader JG. Animals models in research on retinal degenerations: past progress and future hopes. Vision Res 2002;42:393-9.

5 Villegas-Pérez MP, Vidal-Sanz M, Lund RD. Mechanism of retinal ganglion cell loss in inherited retinal dystrophy. Neuroreport 1996;7:1995-9.

6 Villegas-Pérez MP, Lawrence JM, Vidal-Sanz M, et al. Ganglion cell loss in RCS rat retina: a result of compression of axons by contracting intraretinal vessels linked to the pigment epithelium. I Comp Neurol 1998;392:58-77.

7 Wang S, Villegas-Pérez MP, Holmes T, et al. Evolving neurovascular relationships in the RCS rat with age. Curr Eye Res 2003;27:183-96.

8 García-Ayuso D, Salinas-Navarro M, Agudo M, et al. Retinal ganglion cell numbers and delayed retinal ganglion cell death in the P23H rat retina. Exp Eye Res 2010;91:800-10.

9 Wang S, Villegas-Pérez MP, Vidal-Sanz M, et al. Progressive optic axon dystrophy and vascular changes in rd mice. Invest Ophthalmol Vis Sci 2000;41:537-45.

10 García-Ayuso D, Salinas-Navarro M, Agudo M, et al. Retinal ganglion cell axonal compression by retinal vessels in light-induced retinal degeneration. Mol Vis 2011:17:1716-33.

11 Marco-Gomariz MA, Hurtado-Montalbán N, Vidal-Sanz M, et al. Phototoxic induced photoreceptor degeneration causes retinal ganglion cell degeneration in pigmented rats. J Comp Neurol 2006;498:163-79.

12 Vidal-Sanz M, Salinas-Navarro M, Nadal-Nicolás FM, et al. Understanding glaucomatous damage: anatomical and functional data from ocular hypertensive rodent retinas. Prog Retin Eye Res 2012;31:1-27.

13 Sposato V, lovieno A, Sornelli F, et al. Axonal transport deficit in the optic nerve of rats with inherited retinitis pigmentosa and experimentally induced glaucoma. Graefes Arch Clin Exp Ophthalmol 2008;246:1553-8.

14 Pavlidis M, Fischer $D$, Thanos S. Photoreceptor degeneration in the RCS rat attenuates dendritic transport and axonal regeneration of ganglion cells. Invest Ophthalmol Vis Sci 2000;41:2318-28.

15 Eisenfeld AJ, LaVail MM, LaVail JH. Assessment of possible transneuronal changes in the retina of rats with inherited retinal dystrophy: cell size, number, synapses, and axonal transport by retinal ganglion cells. J Comp Neurol 1984; 223:22-34

16 Bourne MC, Campbell DA, Tansley K. Hereditary degeneration of the rat retina. $\mathrm{Br}$ J Ophthalmol 1938;22:613-23.

17 D'Cruz PM, Yasumura D, Weir J, et al. Mutation of the receptor tyrosine kinase gene Mertk in the retinal dystrophic RCS rat. Hum Mol Gen 2000;9:645-51.

18 Gartner S, Henkind P. Pathology of retinitis pigmentosa. Ophthalmology 1982:89:1425-32.

19 Walia S, Fishman GA. Retinal nerve fiber layer analysis in RP patients using Fourier-domain OCT. Invest Ophthalmol Vis Sci 2008:49:3525-8.

$20 \mathrm{Li} Z \mathrm{ZY}$, Kljavin IJ, Milam AH. Rod photoreceptor neurite sprouting in retinitis pigmentosa. J Neurosci 1995;15:5429-38.

21 Newman NM, Stevens RA, Heckenlively JR. Nerve fibre layer loss in diseases of the outer retinal layer. Br J Ophthalmol 1987;71:21-6.

22 Jonas JB, Schiro D. Localized retinal nerve fiber layer defects in nonglaucomatous optic nerve atrophy. Graefes Arch Clin Exp Ophthalmol 1994;232:759-60.

23 Tatham AJ, Weinreb RN, Zangwill LM, et al. Estimated Retinal Ganglion Cell Counts in Glaucomatous Eyes with Localized Retinal Nerve Fiber Layer Defects. Am J Ophthalmol 2013;156:578-87.

24 Zhang $Y$, Xu L, Zhang L, et al. Ophthalmoscopic Assessment of the Retinal Nerve Fiber Layer. The Beijing Eye Study. PLoS One 2013:8:e62022.

25 Bell RD, Zlokovic BV. Neurovascular mechanisms and blood-brain barrier disorder in Alzheimer's disease. Acta Neuropathologica 2009;118:103-13. 